\section{Prácticas de creación análoga-digital mediante la investigación proyectual aplicada a la construcción de personajes para animación}

Jesús Alejandro Guzmán Ramírez ${ }^{(1)}$

\begin{abstract}
Resumen: Mediante la consolidación de un modelo de taller en donde se busca explorar los procesos de creación que decantan en formas de expresión tanto análogas como digitales para procesos de producción animada tridimensional (refiriéndose a este aspecto a ambos ámbitos), se hace un proceso de investigación proyectual que permite consolidar las variables de conformación de personajes que parten de variables aleatorias, pero que van siendo complementadas mediante la indagación, la asimilación de modelos existentes y la amalgama de referentes para llegar al proceso de generación de una matriz que permite el diseño conceptual de un personaje. A partir de este insumo, se empieza un diseño no solo de la estructura en sentido formal del personaje, sino la construcción de su movimiento, personalidad y mecánica, tomando en cuenta las limitaciones y posibilidades que tanto lo análogo como lo digital permiten en el desarrollo y animación de dicho personaje. El proceso es iterativo a lo largo del semestre y busca no solo alimentar una forma de entendimiento proyectual de la construcción animada, sino que busca cuestionar a los estudiantes sobre las tomas de decisiones, las variables que inciden en la factibilidad de un desarrollo que va a ser animado y las soluciones que deben enfrentar para resolver de manera práctica, lo que desde lo narrativo se propone en primera instancia. Como resultado se llega a una producción animada de 5 segundos tanto en análogo como en digital en donde se busca una aplicación práctica de la investigación, así como iniciar el proceso de entendimiento del character design a largo plazo.
\end{abstract}

Palabras clave: Prácticas creativas - estructuras para animación - modelos de producción - sistemas técnicos - sistemas tecnológicos.

[Resúmenes en inglés y portugués en las páginas 134-135]

(1) Doctor en Diseño y Creación. Magister en Diseño y Creación Interactiva, Especialista en video y tecnologías digitales off-line y on-line y Diseñador Visual de la Universidad de Caldas. Realizador Profesional en Animación 3D del Image Campus de Buenos Aires. Profesor Asociado II de la Universidad Jorge Tadeo Lozano. 


\section{Introducción}

El presente texto surge de la construcción, implementación y evolución de la asignatura de Taller II. Estructuras y Modelación, del programa profesional de Realización en Animación de la Universidad Jorge Tadeo Lozano. Dicha asignatura surge en el marco del segundo ciclo de cursos que buscan la consolidación técnica e instrumental de competencias articuladas a los conceptos fundamentales de la animación como forma de representación y proceso audiovisual. Al interior de la asignatura, esta tiene como objetivo "Usar adecuadamente sistemas técnicos y tecnológicos para la construcción de estructuras, volúmenes y andamiajes esenciales para las etapas de preproducción y producción en el diseño de movimiento, en entornos análogos y digitales" (Guzmán-Ramírez, 2019, p. 1). Cuando se habla de diseño, se parte de entender que el programa surge dentro del marco de una escuela de Diseño con gran tradición en Colombia, lo cual no solo influye en la manera en cómo se plantea su sentido conceptual, sino en la dimensión proyectual de cada uno de sus componentes internos.

El concepto de la asignatura parte de los conceptos de técnica, tecnología y sistema tecnológico planteados por Miguel Ángel Quintanilla. Para Quintanilla, la técnica es un grupo de conocimientos o habilidades específicos que permiten resolver problemas de orden práctico, los cuales permiten la generación de artefactos a través de la implementación y sistematización de procesos y la conformación de instrumentos técnicos. A su vez, por tecnología se puede concebir el grupo de conocimientos racionalizados a partir del método científico para describir un problema, explicar un fenómeno, diseñar procesos que responda a cuestionamientos y finalmente aplicar soluciones que desde las técnicas pueden resolver dichos problemas prácticos. Estas últimas, al ser información de carácter cultural, puede ser transmitida bajo las mismas lógicas y formas con las que otros aspectos de las culturas son pasados de generación en generación. Sin embargo, cuando se habla de los artefactos creados, no se piensa en su transmisión sino en su manipulación, modificación o cambio al tener una materialidad física. Cuando se llega al concepto de sistema técnico o tecnológico, se llega a aspectos que involucran dichos artefactos pero que comprenden adicionalmente a los agentes que deben relacionarse con ellos para aplicar una técnica específica a un problema. La diferencia entre sistemas técnicos y tecnológicos, es que en los primeros la relación instrumental entre artefacto y técnica es directa, y a veces depende del aprendizaje vivencial para poder entender su aplicación y formas de apropiación sobre un marco concreto. En el segundo, intervienen además aspectos sociales y organizativos, que llegan a ser tan importantes como los propios artefactos físicos (Quintanilla, 2000, pp. 1-4). Bajo este marco conceptual, la asignatura debía relacionar diferentes tipos de técnicas y tecnologías análogas y digitales para abordar el problema principal del concepto de estructura para personajes de animación en diferentes niveles. Inicialmente, el concepto de estructura y modelación no es visto de manera directa a lo que los términos pueden explicar de manera preliminar, sino que se refiere a los problemas de mecánica del movimiento, las problemáticas técnicas de implementación y los procesos de proyección a corto, mediano y largo plazo que el desarrollo de un rig (esqueleto) pueda tener en una producción animada a partir de la toma de decisiones que se haga sobre la forma en que se generarán los puppets ${ }^{1}$ de stop motion o los rigs ${ }^{2}$ para $C G I^{3}$. 
El tipo de asignatura requería la visión de Tomas Maldonado sobre el concepto del curso básico de la HfG Ulm. En este sentido la necesidad de proveer una introducción a los métodos proyectuales, racionalizar el proceso de diseño de la estructura deseada, e implementar

Los temas de investigación, como estudios de combinatoria y sistemática formal de los productos o investigaciones sobre la complejidad estructural y funcional de los artefactos técnicos. [...] los productos no sólo dieran la apariencia de ser nuevos, sino que realmente se renovaran en su función o en su estructura. La gran preocupación de Maldonado por relacionar las cualidades estructurales y funcionales de los productos, significa que la forma de un producto no ha de ser definida meramente por sus características externas (Bonsiepe et al., 2012, p. 11).

La integración de diferentes tipos de disciplinas se vio como una necesidad, no solo desde la perspectiva del dibujo, sino desde el estudio de anatomía comparada, el manejo de herramientas y materiales en talleres de maderas y metales, así como el aprendizaje de plataformas digitales para animación 3D. Los anteriores tipos de conocimiento se articulan mediante la consolidación de un modelo de taller mixto, en donde se busca explorar los procesos de creación que decantan en formas de expresión tanto análogas como digitales para procesos de producción animada tridimensional (refiriéndose a este aspecto a ambos ámbitos).

\section{Modelo aplicado}

Teniendo claro el concepto de una metodología objetivista experimental vista bajo los postulados de la HfG Ulm aprovechando el concepto del "pensamiento científico operacional” (Bonsiepe et al., 2012, pp. 6-7) y enfocándose en un método proyectual desde el inicio del semestre. Es así como se toman las etapas planteadas desde el modelo académico de la Hfg Ulm como una guía inicial a ser adaptada (Bonsiepe et al., 2012, p. 11):

- Formulación de la tarea.

- Plan de trabajo.

- Fase de información.

- Investigación detallada.

- Desarrollo del producto.

- Fase de decisión y realización.

- Documentación Justificación y descripción del proyecto.

Para lograr una implementación adecuada del concepto de la asignatura, se debía revisar la ruta de prerrequisitos tanto de entrada como de salida del curso para entender, los insumos con los que se cuenta en términos de conocimientos, así como los resultados 
que debe ofrecer para el siguiente nivel en la carrera. En dicho sentido, se tiene el Taller I. Diseño de Movimiento como materia previa, ofreciendo al estudiante un refinamiento claro del sentido del movimiento animado, el entendimiento de las mecánicas y conceptos fundamentales para el desarrollo de la imagen en movimiento desde el dibujo. Las asignaturas posteriores son Fundamentos Análogos 2D, Fundamentos Digitales 2D y Diseño Cinemático y de concepto; las dos primeras se convierten en la aplicación práctica de las lógicas en ambos sistemas de construcción de la imagen animada, con problemas propios de cada tipo de entorno. En algunos casos el procesamiento de nuevas plataformas digitales de construcción animada, que incluyen en algunas ocasiones rigs, y en otros el desarrollo de procesos materiales que implican incluso la generación de plataformas, trucas y sistemas de captura análogo. Para el caso del diseño de concepto, la asignatura de Taller II se ve como un aporte que permite el entender la sistematización para la generación de universos visuales que conformen el mundo con el cual se construye una narrativa basada en un guión.

Teniendo lo anterior como contexto general de la dimensión propia del taller, se quiso buscar una excusa, tanto teórica como procedimental para introducir a los estudiantes a un esquema de construcción mental, que más que simplemente aprender herramientas (sistemas técnicos), dimensionaran las posibilidades, complejidades y relaciones tanto conceptuales como productivas en la generación de las estructuras de tipos de personajes. En primera instancia se ve como una posible problemática de gran escala la introducción de una gran cantidad y variedad de conocimientos en un periodo de 16 semanas, siendo en gran medida el delimitador de los alcances del estudiante. Se estudió el momento de la carrera en la que se ve el taller, y siendo cuarto semestre, es el primer momento totalmente disciplinar, siendo el Taller II el eje principal de carácter teórico-práctico relacionado con la representación de la animación desde la imagen. Adicionalmente la intensidad horaria permite la implementación de metodologías didácticas y pedagógicas acordes a los objetivos propuestos en la materia.

En este punto se vuelve fundamental el concepto de poética como eje base de la idea del taller planteado. En dicha medida, se parte de los postulados de Paul Valery para la idea de la generación de conocimiento a partir de la creación. Paul Valery concibe la poética como un proceso de conocimiento que surge en el hacer de la producción artística (Brigante, 2012, pp. 273-286). Este acto de creación conlleva en su interior procesos no predecibles, propios de la naturaleza vivencial de la que surge en primera instancia, y que está condicionada a la relación del sujeto creador con el resto del colectivo, tanto en sus aspectos positivos como negativos. Estas relaciones vivenciales afectan la concepción de la creación y de los artefactos con las que se conforma, lo que lleva a un cierto "orden" que decanta en la generación misma, que al ser visualizada vincula en este solo acto las reacciones sensibles del individuo (espectador) y la acción humana enfocada en la producción (acto creador). La idea entonces de una creación como un aporte de valor al proceso de aprendizaje, plantea la necesidad de dimensionar la relación valorativa que existe a lo largo del proceso. En esa medida, Valery de manera un poco arbitraria, realiza una analogía a conceptos económicos para contextualizar lo planteado anteriormente. Desde esa perspectiva creador-obra-espectador son llevados a la relación muy cercana al diseño de productor-producto-consumidor (Valéry, 1990, pp. 110-115), que no busca demeritar el carácter viven- 
cial y casi espiritual del proceso creativo en algunas de sus instancias, sino que habla de la apropiación que se hace de referentes visuales, materiales, culturales y cognitivos en un momento histórico, el cual posee cánones pragmáticos para determinar la importancia de diversos componentes del marco de la realidad, y en donde lo sublime es mucho más sutil y difícil de definir.

En el proceso de dar valor a la obra, el creador cierra su relación con esta al entregar todo un proceso de investigación, desarrollo técnico y aplicación para la generación del "producto", finalizando en términos prácticos cuando el creador se desprende del artefacto. La culminación del ciclo por parte del artista significa el inicio del ciclo en otro extremo que es el de la audiencia (consumidores), que al ser entregada al colectivo sin saber qué rumbos tomará dicha creación, se resignifica y es "modificada" en su valía por la sociedad que de una u otra manera dio vida a dicha obra, tal como lo cita Valéry:

Únicamente podemos considerar la relación de la obra con su productor, o bien la relación de la obra con aquel a quien ella modifica una vez realizada. La acción del primero y la reacción del segundo no pueden confundirse nunca. Las ideas que uno y otro se hacen de la obra son incompatibles (1990, p. 113).

Este aspecto es muy importante para el programa de Realización en Animación, pues se busca que el egresado tenga conciencia de que su producción debe estar enlazada a un público objetivo, el cual debe ser analizado previamente y entendido no solo desde la audiencia sino desde los circuitos de divulgación y difusión de su producto final.

En el proceso de implementación de estos conceptos al interior de la asignatura, se pudieron evidenciar elementos que permiten establecer una relación directa entre esta visión de la poética (poiética) con los postulados abordados por Alain Findeli (Findeli, Brouillet, Martin, Moineau, \& Tarrago, 2008, pp. 70-72) alrededor de los tipos de investigación en diseño. De esta manera, al buscar un equiparamiento entre los conceptos planteados desde la revisión teórica alrededor de la poética y la revisión de enunciados que son propios del proceso de diseño, se llega a una relación directa a la que se puede hacer mención (ver Tabla 1) (Guzmán Ramírez, 2018, pp. 153-154. En F. C. Londoño).

\begin{tabular}{|l|l|}
\hline Diseñador & Enunciador, creador, productor \\
\hline Diseño & Imagen, obra, producto \\
\hline Usuario & Enunciatario, receptor, espectador \\
\hline
\end{tabular}

Tabla 1. Relación entre diseño y la visión de poética. Fuente: El autor. 2019.

El uso y comprensión de elementos originados en el arte, la necesidad constante de entender la realidad que circunscribe a usuarios, artefactos y usos, así como una verdadera articulación de sistemas tecnológicos dentro de la conformación discursiva de la creación, 
evidencia el valor inmanente que el diseño posee como una forma de abordaje concebida en el hacer.

A partir de lo anterior se puede ver como la creación en sí misma combina deseos, materia e individuos en una sola elaboración (Valéry, 1990, pp. 124-128). Es así como se puede decir que el creador muere en su estado previo, debido a que es modificado por la experiencia generadora a través de las vivencias y concepciones que mutaron mientras eran convertidas en representación. Posterior a esta "muerte" emerge un nuevo sujeto a través de su producción (relación temporal ser-objeto (Montoya Santamaría, 2011, pp. 69-76; Sanín Santamaría, 2006, p. 61)).

Retomando el concepto de la excusa temática se hace un proceso de investigación proyectual que permite consolidar las variables de conformación de personajes que parten de selecciones aleatorias (ver Imagen 1), pero que van siendo complementadas mediante la indagación, la asimilación de modelos ya existentes en la naturaleza y la amalgama de referentes para llegar al proceso de generación de una matriz que permite el diseño conceptual de un personaje. En este punto, tanto la anatomía comparada, el uso de sistemas de lógica relacional, así como el uso de ciertos aportes teóricos se vuelven fundamentales para que el estudiante comprenda la dimensión compleja de la construcción de una criatura, adicional al hecho de que normalmente se encuentran alejados de la visión del bípedo humano tradicional. Para reflejar el concepto que se tiene de la generación de un ser se usa como texto base en el primer corte del semestre (4 semanas) el documento llamado "Complejidad e incertidumbre. Nuevas ideas para la inteligibilidad de las formas vivas" de Jorge Wagensber (Wagensberg, 1999). Este texto permite entender la complejidad en relación entre la generación de las especies y los contextos en los que se desarrolla, así como la evolución que variables de incertidumbre tienen sobre la evolución de diferentes especímenes en la naturaleza, así como su comportamiento y sistemas de adaptación.

Para el proceso proyectual de la asignatura Taller II se asumen los aspectos propios de los esquemas de industrialización en los que ha entrado el sector de la animación desde hace décadas. Aunque existen muchas etapas en el desarrollo de una producción animada que son de carácter artesanal si se quiere, se busca que mediante las metodologías del diseño se puedan planificar, dimensionar, ejecutar y resolver los elementos prácticos que requiere la creación que se ha concebido en la etapa de asignación aleatoria del personaje. Para ello se hace un proceso de indagación e investigación proyectual, que busca determinar las características, esquemas y prototipos de la criatura que se está elaborando, para tener elementos de juicio al entrar en la etapa de producción (ver Imagen 2).

La matriz que se presenta se divide en seis secciones que permiten la evolución del proyecto de personaje durante las primeras cuatro semanas del semestre. En la primera sección se registra el resultado de la tabla automatizada de personajes, al igual que una sinopsis del personaje basado en el texto de Jorge Wagensberg. Esta etapa permite dimensionar el contexto del personaje, su origen y los elementos mínimos que lo componen. En la segunda sección se llena un cuadro que refina las características del personaje a partir de arquetipos de personalidad, características morfológicas específicas, formas de diseño básicas y un concepto del comportamiento que se espera tenga el personaje. Esta etapa define las características formales de la creatura, pero también las lógicas mecánicas con las que se va a desplazar el mismo. En la tercera sección se genera un esquema conceptual 


\begin{tabular}{|c|c|c|c|}
\hline Estructura Primaria & Extremidades adicionales & Apendices & Estructura secundaria \\
\hline Reptante & Ninguna & Ninguno & Reptante \\
\hline Acuático & Dos & Cola & Acustico \\
\hline Cuadrúpedo & Cuatro & Cuernos & Cuadrúpedo \\
\hline Alado & & Segunda cabera & Alado \\
\hline Bipedo & & Caparazón & Bipedo \\
\hline & & Escamas prominentes & Ninguno \\
\hline Bipedo & Cuatro & Segunda cabera & Acustico \\
\hline
\end{tabular}
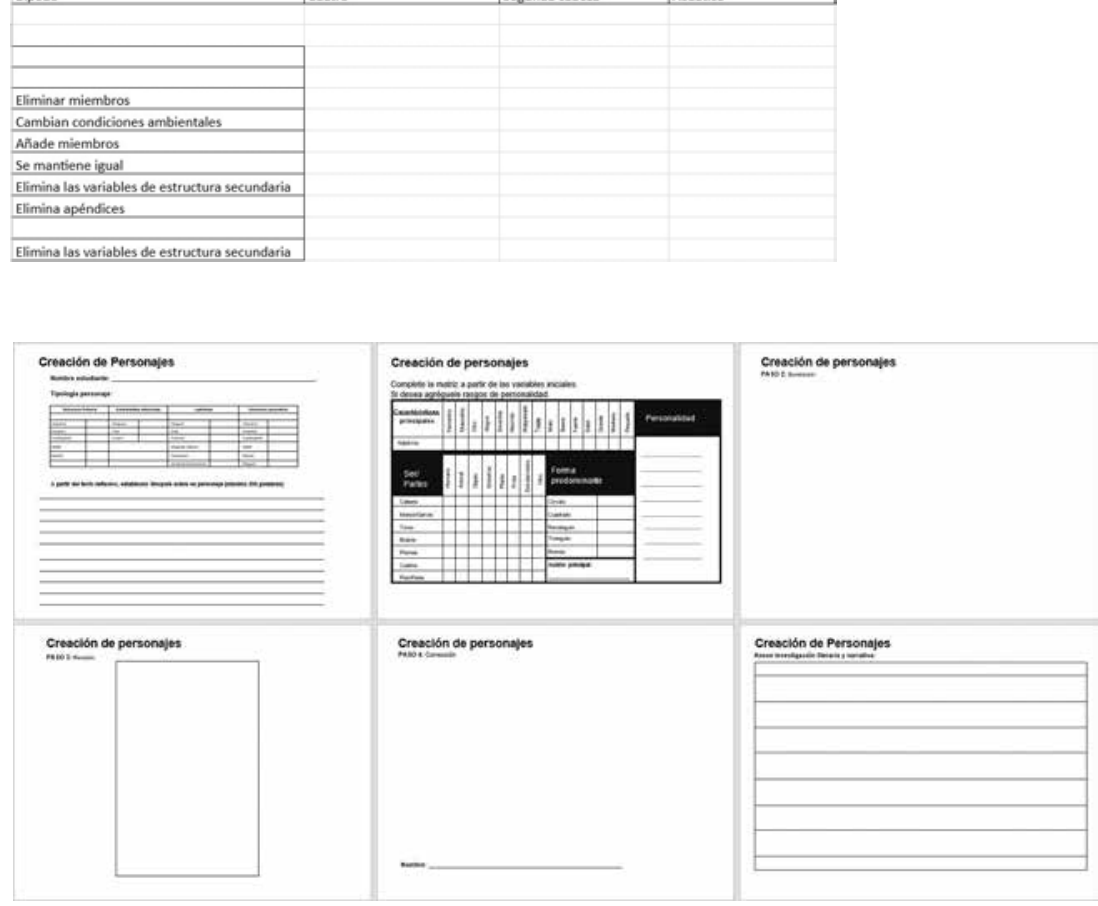

Imagen 1. Tabla automatizada de generación de personajes no humanoides. Llamada coloquialmente en el Taller II como el “Generador dela vida”. Fuente. El autor. Imagen 2. Matriz de creación de personaje. Fuente: El autor.

a nivel de boceto, que integra forma general y sistema de estructura interna del personaje, pensando en articulaciones y formas de movimiento. La cuarta sección se utiliza a manera de sistema de coevaluación, en donde un compañero toma las tres primeras secciones de la matriz, las analiza y sugiere o plantea interrogantes frente a las resoluciones visuales que se presentan en relación con los elementos conceptuales de la investigación. En la quinta sección el estudiante realiza correcciones planteadas por el compañero y el docente para un refinamiento del personaje en todo sentido y en la sexta sección se inserta todo el proceso 
de investigación literaria y narrativa que se tuvo a lo largo del primer corte para sustentar el resultado final de esta primera etapa.

A la par el estudiante va teniendo prácticas en programa 3D digitales para comenzar a interpretar las lógicas de animación vistas hasta el momento en la carrera en un entorno diferente al trabajado desde el dibujo animado. Igualmente se trabaja en los conceptos básicos de programación orientada a objetos para comprender relaciones de estructuras, controles y resultados. Desde lo análogo se están realizando prácticas con papel, madera y alambre para ejecutar y resolver problemas relacionados con los diferentes grados de libertad de una coyuntura, análisis de materiales y respuesta de los mismos al problema del movimiento.

Como se puede ver, esta primera etapa tiene todos los aspectos fundamentales del diseño en cuanto a la reconstrucción de la realidad mediante la proyección, representación y esquematización de las problemáticas presentadas, para de manera iterativa ir determinando el mejor sistema de abordaje desde el punto de vista productivo. Es así como se puede citar el texto de Modelos de Ulm:

Diseñar es una actividad que está orientada a la industria, a la tecnología y al trabajo interdisciplinario. Situaciones complejas y los problemas que resultan de ellas son el campo en el cual el diseñador se desempeña, ya sea creando productos, gráficos o información visual. La creciente complejidad de estas circunstancias hizo indispensable la introducción de materias y modos de pensamiento científicos en el diseño. Impulsos fundamentales para ello vinieron de las matemáticas, de los procedimientos de programación para sistemas de computadoras y de las técnicas de planificación y organización. De este contexto emergió la teoría de los procedimientos proyectuales, la así denominada Metodología del Diseño (Bonsiepe et al., 2012, p. 10).

\section{El acting como práctica creadora}

Uno de los aspectos más valiosos, pero casi invisibles del proyecto del Taller II es el proceso de acting y caracterización de personaje. En dicha medida se ha dado a lo largo de la carrera sesiones de acting e interpretación que soportan el entendimiento de la proyección del animador hacia el personaje. En el primer semestre se plantea un entendimiento y conciencia del cuerpo desde postulados cercanos a la biomecánica de Vsévolod Meyerhold, en segundo semestre se usa el concepto del "sí mágico" de Konstantín Stanislavski para la generación de personajes realistas, y en el Taller II (cuarto semestre) se usan los postulados de Jacques Lecoq para la construcción de personajes fantásticos. Todo esto se usa como insumo para la dimensión artificial del personaje que se va a crear en pos de ser vistos por un posible espectador.

Cuando se hace referencia al concepto de lo artificial, no se habla de la acepción de un elemento externo a los individuos, sino que por el contrario, se refiere a una dimensión de las cosas que tienen su origen en lo humano, y que más allá de su materialidad o de su forma 
de uso, se relaciona con los por qué existe un artefacto o un sistema artificial, el cómo se hizo, por quiénes y con qué objetivo (Krippendorff, 2007, p. 17). Esto habla directamente de la cultura de un colectivo y de la forma en que se desarrolla un hacer específico. $\mathrm{Al}$ relacionar este tipo de dimensión al fenómeno animado, se debe partir de encontrar puntos de construcción de estos discursos colectivos alrededor del cómo surge este sistema cultural de apropiación del movimiento pensando siempre en que va a ser observado por otros. En este sentido existe una metáfora desde el teatro que habla explícitamente de una relación entre el artefacto narrativo y el sistema tecnológico con el que se implementa esa apropiación de ideas para convertirse en movimiento. Eugenio Barba logra hacer un vínculo entre las narraciones y la construcción dramatúrgica teniendo como eje el concepto del texto. Para Barba "la palabra 'texto', antes que significar un texto hablado o escrito, manuscrito o impreso significa 'tejido' (...) Lo que concierne al 'texto' (al tejido) del espectáculo, puede ser definido como 'dramaturgia'; es decir drama-ergon, 'trabajo', 'obra de las acciones”" (Barba \& Savarese, 2010, p. 73). En este solo modo de expresión, Barba no solo relaciona una relación directa entre el concepto de acción con el movimiento o la obra, sino que expresa de manera muy sublime a través del tejido una de las funciones que se han planteado alrededor de la poética a lo largo de la implementación en el aula de clase.

Al hablar de tejido, de sistema de enlazamiento o encadenamiento entre las narraciones e historias de un posible espectador y las formas de apropiación a través de la acción que surgen en lo dramatúrgico, Barba cohesiona la función propia del artista que obtiene un insumo del colectivo, lo vivencia, modifica y a través de la acción creadora emerge como una obra que vuelve a ser parte de dicho colectivo pero en forma de expresión corporal que interpreta realidades y sentires de los espectadores. De igual manera, al hablar de la obra de las acciones sobre este tejido, se refiere a una difusa forma de poder del actor sobre el relato y sus personajes, equiparables a lo que hace el animador sobre el cuerpo animado. Esta forma de poder ejerce agencia creadora a manera de terceridad sobre un artefacto (personaje teatral o animado) con potencial de movimiento y narración.

Las formas del hacer en la animación no se encuentran tan lejanas de los componentes formales del teatro, tanto en su esencia como en las lógicas con las que se llega a la creación poética del movimiento. Es más, se pueden describir fácilmente puntos de encuentro entre las estructuras conceptuales de la actuación y de la interpretación del animador. Lo anterior se evidencia a través de las diversas prácticas que se pueden encontrar en el cruce entre estos dos saberes como por ejemplo cuando se realiza un dibujo animado, se manipula una figura en stop motion o en 3D, se realiza una puesta en escena de un personaje en medio de una obra teatral o se juega con los hilos que dotan de vida a una marioneta. Todos los aspectos mencionados describen de una u otra manera un sistema artificial de vínculos "físicos", si así se quiere, entre el ejecutante con el artefacto de ejecución, es decir el personaje, siendo dotado de una vida impuesta por el actor-animador, pero que, como origen intencional del movimiento, este actor-animador se desvanece frente al espectador pues este es atrapado por la "máscara" que resulta ser el escenario o la pantalla.

En este sentido, tanto actor como animador son definidos en primera instancia por una necesidad de entendimiento técnico del cuerpo, para que una vez identificada cada una de sus partes, exista una conciencia de sus posibilidades y limitaciones. Ambos, una vez 
inmersos en una lógica biomecánica, pueden definir los elementos fundamentales del diseño de la mecánica, forma y ánima de un personaje, que en este caso se convierte en la obra de creación en sí misma, y la interpretación es el acto creador que usa las acciones como sistema tecnológico. Dichas acciones son construidas, diseñadas y utilizadas de manera sistémica con un fin, al usar el cuerpo como materia de insumo que se modifica ya sea para generar el personaje en escena o para ser aplicado sobre el tipo de mediación técnica que requiera el animador.

Tanto acciones corporales en un teatro, como dispositivos de captura o acetatos de dibujo, son agentes no humanos que ejercen una agencia delegada sobre una construcción artificial que es el personaje, siendo todos en cierto modo objetos creados previamente desde el movimiento al nivel de la pre-expresividad. Estos agentes no humanos exponen la intención del agente humano, en este caso el actor-animador, a través del diseño de la forma mediante el proceso iterativo de ensayo y error, expresándose a través de estos y delegando en la creación final depurada del movimiento la función de interrelación con el espectador, para iniciar de esta manera el ciclo de conformación de creación poética que debe ser asumida por el colectivo (Latour, 2001, pp. 208-231). La diferencia sustancial entre este proceso de construcción del movimiento y lo referente a la cotidianidad de un individuo se refiere a la activación consciente de lo que se mueve, cómo se mueve y para qué se mueve. En lo cotidiano, el movimiento es parte de un sistema casi "automatizado" que es inherente al humano desde su propio $\mathrm{ADN}$, y en donde la relación con el espacio ha sido un desarrollo instintivo que ha definido el sentido de humanidad. Sin embargo, lo que se plantea como un acto creador expresivo, involucra no solamente la toma activa de la consciencia del movimiento del artista, sino la relación de lo que comunica hacia el espectador.

Barba hace uso de un concepto similar al de agentes humanos y no humanos de Latour y lo describe como "acciones" (2010, p. 74), las cuales son componentes fundamentales de la dramaturgia. Estas las define como una expresión latente en el movimiento que se construye, que es finalmente lo que incide de manera directa sobre las expectativas de quien observa a un personaje o una puesta en escena, modificando en mayor o menor medida su entendimiento de la realidad planteada artificialmente, así como de la que concibe como propia, incidiendo en la modificación que se logre de la percepción que se posee en adelante de ambas realidades.

Todos estos conceptos deben ser interpretados por el estudiante para concebir no solo el movimiento que va a tener el personaje, sino sus particularidades y cómo estas van a incidir en la generación de las estructuras tanto análogas como digitales y los sistemas de producción que se van a involucrar en el desarrollo del semestre.

\section{Mecánica y estructuras}

A partir del insumo generado desde la investigación literaria y narrativa, el diseño conceptual del personaje y la construcción de la caracterización e interpretación del movimiento del mismo, se empieza un diseño no solo de la estructura en sentido formal del personaje, 
sino la construcción de su movimiento, personalidad y mecánica, tomando en cuenta las limitaciones y posibilidades que tanto lo análogo como lo digital permiten en el desarrollo y animación de dicho personaje.

La segunda etapa se enfoca en el desarrollo mecánico del trabajo, teniendo como referente teórico el capítulo de Movimiento del libro Mechanization Takes Command - A Contribution to anonymous history de Siegfried Giedieon (1970, pp. 14-34). Este texto busca que los estudiantes comprendan que el estudio del movimiento es algo que se ha desarrollado tanto desde el método científico como desde la representación visual, y tiene sentido en la medida que puedan transpolar estos principios a los esquemas que van generando para el desarrollo de la propuesta de personaje que han ido construyendo a lo largo del semestre. El proceso es iterativo a lo largo de las 16 semanas y busca no solo alimentar una forma de entendimiento proyectual de la construcción animada, sino que pretende cuestionar a los estudiantes sobre las tomas de decisiones, las variables que inciden en la factibilidad de un desarrollo que va a ser animado y las soluciones que deben enfrentar para resolver de manera práctica, lo que desde lo narrativo se propone en primera instancia. Como resultado se llega a una producción animada de 5 segundos tanto en análogo como en digital en donde se busca una aplicación práctica de la investigación, así como iniciar el proceso de entendimiento del character design a largo plazo.

A continuación, se deja un enlace con algunos resultados finales del proceso: https://drive. google.com/drive/folders/15AXEzExC_LAXnQ7Jm9yqpz-nCAxnwzwS?usp=sharing

\section{Notas}

1. Se hace uso desde el inglés al término puppet de manera estándar en el sector de la animación para referirse a los muñecos de stop motion.

2. El concepto de rig es un término inglés que significa andamiaje. En el Sector industrial de la animación digital, es la manera en la que se cita este tipo de "esqueleto" combinado con programación.

3. CGI es la sigla de Computer Graphic Image.

\section{Referencias Bibliográficas}

Barba, E. \& Savarese, N. (2010). El arte secreto del actor: Diccionario de antropología teatral. (4th ed.). Lima: Editorial San Marcos E.I.R.L.

Bonsiepe, G.; Bürdek, B. E.; Fernández, S.; Hausmann, B.; Walther, E.; von Seckendorff, E.;... Huff, W. S. (2012). Modelos de Ulm, Modelos Post Ulm / hochschule für gestaltung Ulm 1953 - 1968. Retrieved from http://www.catedranaranja.com.ar/biblioteca/documentos/ notas_apuntes/MODELOS_DE_ULM.pdf

Brigante, A. M. (2012). La Teoría de la Acción Poética de Paul Valéry. Pensamiento, 68(256), 273-286. 
Findeli, A.; Brouillet, D.; Martin, S.; Moineau, C. \& Tarrago, R. (2008). Research Through Design and Transdisciplinarity: A Tentative Contribution to the Methodology of Design Research. «FOCUSED»- Current Design Research Projects and Methods, 67-91. Retrieved from http://swissdesignnetwork.ch/src/publication/focused-current-design-research -projects-and-methods-2008/SDN-Publication-2008_Focused.pdf\#page $=69$

Giedion, S. (1970). Mechanization Takes Command - A Contribution to anonymous history (3rd ed.). New York: Oxford University Press.

Guzmán-Ramírez, J. A. (2019). Facultad de Artes y Diseño| Unidad Académica: Programas de Diseño Visual | Programa: Realización en Animación [Taller 2: Estructuras y Modelación]. Bogotá.

Guzmán Ramírez, A. (2018). Aportes a la Poética (Poietica) desde la Sociología de la Ciencia y el Constructivismo Social. In F. C. Londoño, A. Gómez Alzate, A. L. Grisales, \& G. M. Mejía (Eds.), Diseño y Creación Foro Académico Internacional 17 festival Internacional de la Imagen. Nuevas expediciones. Universidad de Caldas.

Krippendorff, K. (2007). An Exploration of Artificiality. Artifact, 1(2), 17-22. https://doi. org/10.1080/17493460600610848

Latour, B. (2001). la esperanza de pandora - Ensayos sobre la realidad de los Estudios de la Ciencia (1st ed.). Barcelona: Editorial Gedisa.

Montoya Santamaría, J.W. (2011). Tecnociencia y racionalidad en el mundo contemporáneo. Trilogía - revista ciencia, tecnología y Sociedad, (4), 69-76.

Quintanilla, M. Á. (2000). Técnica y cultura. Teorema, 17(3), 1-16. Retrieved from http:// www.oei.es/salactsi/teorema03.pdf

Sanín Santamaría, J. D. (2006). ESTÉTICAS DEL CONSUMO Configuraciones de la cultura material. Universidad Nacional de Colombia, Medellín.

Valéry, P. (1990). Teoría poética y estética. Retrieved from https://ia902709.us.archive.org/25/ items/ValeryPaulTeoriaPoeticaYEsteticaOCR/Valery-Paul-Teoria-poetica-y-estetica -OCR.pdf

Wagensberg, J. (1999). Complejidad e incertidumbre. Nuevas ideas para la inteligibilidad de las formas vivas. Mundo Científico, (201), 41-60.

\begin{abstract}
Through the consolidation of a workshop model that seeks to explore the creation processes that opt for both analog and digital forms of expression for three-dimensional animated production processes (referring to this aspect in both areas), a project research process is carried out that allows to consolidate the variables of conformation of characters that start from random variables, but that are being complemented by means of the investigation, the assimilation of existing models and the amalgamation of referents to arrive at the process of generating a matrix that allows the conceptual design of a character. From this input, a design begins not only of the structure in the formal sense of the character, but the construction of its movement, personality and mechanics, taking into account the limitations and possibilities that both analog and digital allow in development. and animation of said character. The process is iterative throughout the semester
\end{abstract}


and seeks not only to feed a form of projective understanding of animated construction, but also seeks to question students about decision-making, the variables that affect the feasibility of a development that will be encouraged and the solutions they must face to solve in a practical way, which from the narrative is proposed in the first instance. As a result, an animated production of 5 seconds is reached, both in analogue and digital, where a practical application of the research is sought, as well as starting the process of understanding character design in the long term.

Keywords: Creative practices - animation structures - production models - technical systems - technological systems.

Resumo: Através da consolidação de um modelo de oficina que visa explorar os processos de criação que optam por formas de expressão analógicas e digitais para processos de produção de animação tridimensional (referindo-se a este aspecto em ambas as áreas), é realizado um processo de investigação de projetos que permite consolidar as variáveis de conformação de caracteres que partem de variáveis aleatórias, mas que se complementam por meio da investigação, da assimilação de modelos existentes e do amálgama de referências para chegar ao processo de geração de uma matriz que permita o desenho conceitual de um personagem. A partir dessa entrada, parte-se de um desenho não só da estrutura no sentido formal do personagem, mas da construção de seu movimento, personalidade e mecânica, levando em consideração as limitações e possibilidades que tanto analógicas quanto digitais permitem no desenvolvimento. $\mathrm{E}$ animação de disse personagem. $\mathrm{O}$ processo é iterativo ao longo do semestre e visa não só alimentar uma forma de compreensão do projeto da construção animada, mas também questionar os alunos sobre a tomada de decisão, as variáveis que afetam a viabilidade de um empreendimento que se pretende fomentar e as soluções que devem enfrentar para resolver de forma prática, o que se propõe a partir da narrativa em primeira instância. Como resultado, chega-se a uma produção animada de 5 segundos, tanto em analógico como digital, onde se procura uma aplicação prática da investigação, bem como se inicia o processo de compreensão do design de personagens a longo prazo.

Palavras chave: Práticas criativas - estruturas de animação - modelos de produção - sistemas técnicos - sistemas tecnológicos.

[Las traducciones de los abstracts fueron supervisadas por el autor de cada artículo] 